This work is under IEEE copyright and its IEEE published version may be found in http://www.ieeexplore.ieee.org. This file constitutes the draft version. Work retroactively supported by project TEC2013-47020-C2-2-R (Ministerio de Economia y Competitividad - SPAIN), published in 2014 before the project's official awarding date

Presented in 2014 IEEE International Conference on Acoustics, Speech and Signal Processing (ICASSP), Florence (Italy), April 2014.

DOI: $10.1109 /$ ICASSP.2014.6854709

\title{
FREQUENCY-DOMAIN GLR DETECTION OF CYCLOSTATIONARY SIGNALS IN FREQUENCY-SELECTIVE CHANNELS
}

\author{
Josep Font-Segura, Jaume Riba, Javier Villares and Gregori Vazquez
}

\author{
Signal Theory and Communications Department, Technical University of Catalonia (UPC) \\ D5-\{214, 116, 115, 204\}, Campus Nord UPC, Jordi Girona 1-3, 08034 Barcelona, Spain \\ Email: \{josep.font-segura, jaume.riba, javier.villares, gregori.vazquez\}@upc.edu
}

\begin{abstract}
The frequency diversity exhibited by cyclostationary signals is exploited in this paper. A novel rank-1 frequency-domain representation of a digital waveform is proposed to address the generalized likelihood ratio (GLR) detection of a cyclostationary signal with unknown white noise. With the aim of avoiding the well-known sensitivity of cyclostationary-based detectors to frequency-selective fading channels, a parametric channel model based on the coherence bandwidth is adopted and incorporated in the GLR test. The proposed detector outperforms the classical spectral correlation magnitude detectors by exploiting the rank-1 structure of small spectral covariance matrices.
\end{abstract}

Index Terms- GLRT, LMPIT, cognitive radio, cyclostationarity based detection, spectral correlation.

\section{INTRODUCTION}

Reliable primary users (PU) signal detection under challenging situations such as low signal-to-noise ratio (SNR), shadowing and fading is a key enabling factor in interweave cognitive radio [1]. This has motivated recent attention on the specific problem of PU signal detection in white noise. A variety of detection methods have been proposed for several degree of side information: while enhanced capabilities are achieved with higher degrees of side information, these methods suffer from higher sensitivity to model inaccuracies.

The adoption of the PU model is a relevant issue, and may include features such as the transmitted power spectral density [2], cyclostationarity, modulation type, etc. [1]. On the other hand, the frequency-selective and time-varying nature of the wireless propagation channel can severely affect the detectors' performance [3]. The use of multiple antennas has received attention in order to improve performance [4-7]. The multiple antenna formulation leads to low rank spatial

This work has been partially funded by the Spanish Government under TEC2010-21245-C02-01 (DYNACS), CONSOLIDER INGENIO CSD200800010 (COMONSENS), CENIT CEN-20101019 (THOFU), and the Catalan Government (AGAUR) under Grant 2009SGR1236 and Fellowship FI-2010. covariance matrices. This fact is exploited to improve, for instance, the noise floor estimation, as one has access to a noise subspace which is free of the signal component. In the single antenna case, the specification of a model for the temporal autocorrelation function of PU and noise components is required. By resorting to asymptotic properties of Toeplitz matrices, [8] shows that detection can be formulated from the signal periodogram, smoothed by an appropriately selected spectral mask. This yields to significant saving on computational complexity, which is specially interesting in order to make cognitive radios feasible.

This paper formulates the single-antenna detection problem of a second-order cyclostationary signal by taking benefit from the mature and recent detection theory advances in array processing. This provides an original formulation of cyclostationary detection based on a vectorial frequency-domain processing that leads to a signal model which exhibits low rank structure. Particularly, the rank-1 structure for pulse-shaped digital modulations considered in our formulation plays the equivalent role of the spatial signature typically found in the array processing field.

Cyclostationarity feature detection had originally emerged as a tool to relax the assumptions on the noise statistics [9], and it has hence gained recent attention in the field of spectrum sensing for weak signals $[10,11]$ and unknown noise statistics $[7,11,12]$ to achieve robustness to SNR walls [13]. In particular, the scheme reported in [10] and method based on $[6,11]$ are considered as benchmarking. The frequencydomain formulation adopted in this work was introduced by the authors in [14] in the SNR estimation problem.

\section{PROBLEM FORMULATION}

This work addresses the detection of a digital pulse-shaped modulated signal as

$$
\begin{aligned}
& \mathcal{H}_{0}: y(t)=n(t) \\
& \mathcal{H}_{1}: y(t)=n(t)+x(t),
\end{aligned}
$$

where $n(t)$ is a wide sense stationary (WSS) circular noise of power spectral density (PSD) $N_{0} / 2$ inside the band of inter- 
est, and $x(t)$ is given by

$$
x(t)=\sqrt{\gamma T} h(t) * \sum_{n} a[n] p(t-n T) .
$$

In (2), $\gamma>0$ is the signal strength, $T$ is the symbol interval, $h(t)$ is the complex propagation channel, $a[n]$ is the unit variance symbol sequence, and $p(t)$ is the unit energy modulation pulse. This signal model is valid for a wide class of digital modulations such as pulse amplitude modulation (PAM), quadrature amplitude modulation (QAM) and amplitude phase shift keying (APSK). The two-sided bandwidth of $x(t)$ is $B=(1+\alpha) / T$, where $0<\alpha<1$ is the excess bandwidth or roll-off parameter.

\subsection{Cyclostationary Signal Background}

This section summarizes the main concepts behind cyclostationarity. The reader is referred to [15-19] for more details on this subject. The cyclic spectral density (CSD) of a process $x(t)$ is defined as the cross spectral density $S_{x}^{\alpha}(f) \doteq$ $\lim _{T_{0} \rightarrow \infty} E\left[X_{T_{0}}\left(f+\frac{\alpha}{2}\right) X_{T_{0}}^{*}\left(f-\frac{\alpha}{2}\right)\right]$, where $X_{T_{0}}(f)$ is the normalized finite-size Fourier transform of a $T_{0}$-size realization of the process. Assuming stationary zero-mean uncorrelated symbols, the CSD of (2) is given as

$$
S_{x}^{l / T}(f)=\gamma G\left(f+\frac{l}{2 T}\right) G^{*}\left(f-\frac{l}{2 T}\right),
$$

for $l \in \mathbb{Z}$ and 0 for $l \notin \mathbb{Z}$, and $G(f)$ is

$$
G(f)=H(f) P(f)
$$

i.e., the product of the channel frequency response and the modulation pulse Fourier transform. The factorization (3) holds even in the presence of a frequency-selective channel, as it is consequence of the pulse-shaped modulation structure.

\subsection{Asymptotic Frequency-Domain Signal Detection}

Now, consider the Fourier transform of $M$ blocks of length $T$ of $y(t)$, namely, $Y_{M T}(f)$. In vector notation, the frequencydomain observation vector is defined as

$$
\mathbf{y}(v) \doteq Y_{M T}(\mathbf{f}(v))
$$

where $\mathbf{f}(v)$ scans the sensing interval $(-B / 2, B / 2)$ with small sampling intervals of $L \doteq\lceil B T\rceil$ samples through the auxiliary variable $-1 /(2 T) \leq v \leq 1 /(2 T)$, i.e.,

$$
\mathbf{f}(v)=v \mathbf{1}+\underbrace{\frac{1}{T}\left(\begin{array}{c}
(L-1) / 2 \\
\vdots \\
-(L-1) / 2
\end{array}\right)}_{\dot{\mathrm{s}}} .
$$

Under this notation, a consequence of (3) and the stationarity of the noise is that the second-order statistics of (5) obey

$$
\mathbb{E}\left[\mathbf{y}(v+\delta) \mathbf{y}^{H}(v)\right] \rightarrow\left\{\begin{array}{cc}
\mathbf{S}_{\mathbf{y}}(v) & \delta=0 \\
\mathbf{0} & \delta \neq 0
\end{array},\right.
$$

where the entries of the $L \times L$ spectral matrix $\mathbf{S}_{\mathbf{y}}(v)$ are related to the CSD of $y(t)$ by

$$
\left[\mathbf{S}_{\mathrm{y}}(v)\right]_{i, j \in\{1, \ldots, \mathrm{L}\}}=S_{y}^{f_{i}(v)_{i}-f_{j}(v)}\left(\frac{f_{i}(v)+f_{j}(v)}{2}\right),
$$

where $f_{i}(v)$ is the $i$-th element of $\mathbf{f}(v)$. By introducing a vectorial spectral process to treat cyclostationary signals, the uncorrelation property (7) is hereby unveiled. In fact, this constitutes and extension of the frequency-domain treatment of WSS signals to cyclostationary signals, just by extending the dimension of the frequency-domain process.

It is a well-known result that for a fixed value of $f$, $X_{T_{0}}(f)$ has asymptotic normality as $T_{0} \rightarrow \infty$ [20]. As a result of, the log-likelihood function of the frequency-domain observation vector (5) can be written as

$$
L(\mathbf{y})=-\int_{1 / T}\left(\ln \operatorname{det}\left(\mathbf{S}_{\mathbf{y}}(v)\right)+\mathbf{y}^{H}(v) \mathbf{S}_{\mathbf{y}}^{-1}(v) \mathbf{y}(v)\right) d v .
$$

An advantage of this formulation is that the matrix involved in (9) exhibits low dimensionality $L$, independent from the data size. Hence, mathematical and computational simplicity will be attained compared with the time-domain approach which typically involves the estimation of cumbersome covariance matrices, e.g., as in [21]. Using (3), the spectral matrices under both hypotheses read

$$
\begin{aligned}
& \mathcal{H}_{0}: \quad \mathbf{S}_{\mathrm{y}}(v)=N_{0} \mathbf{I}, \\
& \mathcal{H}_{1}: \quad \mathbf{S}_{\mathbf{y}}(v)=N_{0} \mathbf{I}+\gamma \mathbf{g}(v) \mathbf{g}^{H}(v),
\end{aligned}
$$

where $\mathbf{g}(v)=G\left(\mathbf{f}_{v}\right) \odot e^{j 2 \pi \varepsilon \mathbf{s}}$, being $0 \leq \varepsilon \leq 1$ the timedelay or timing parameter.

It is noted that as a result of the frequency-domain formulation of the likelihood function, the general problem posed in (1) is translated to the specific problem (10). Here, the important feature of (10) is that the signal component has rank-1 structure, while the noise component is full-rank. This lowrank nature of the signal to be detected is what provides the basis of the detection of cyclostationary signals developed in the sequel.

\section{GLR DETECTION OVER FREQUENCY-SELECTIVE FADING CHANNELS}

It is known that in the presence of unknown frequencyselective channels, the performance of cyclostationary-based detection methods is severely downed [3]. This is due to the fact that the channel can attenuate or even kill the specific cyclostationary feature which is exploited for detection. 
However, as stated in the former section, pulse-shaped modulated signals preserve the factorization property (3) even in frequency-selective channels. This means that the rank-1 structure is still present in the received waveform as indicated in (10), but with a modified pulse shaped according to (4). Under the asymptotic frequency-domain formulation, this is modeled as

$$
\mathbf{g}(v)=\sqrt{g(v)} \tilde{\mathbf{g}}(v),
$$

i.e., $g(v) \doteq\|\mathbf{g}(v)\|$ models the frequency-dependent arbitrary gain, $\tilde{\mathbf{g}}(v)$ is a unit-norm vector. If the coherence bandwidth of the channel is known in the form $B_{c}=1 /(K T)$, a $K$-parameterization of (11) is proposed as

$$
\mathbf{g}(v)=\sqrt{g_{k}} \tilde{\mathbf{g}}_{k},
$$

for $v \in I_{k}$, where

$$
I_{k}=\left(\frac{-1 / 2+(k-1) / K}{T}, \frac{-1 / 2+k / K}{T}\right),
$$

for $k=1, \ldots, K$. Note that the key assumption in the previous model is that every entry of the frequency vector (12) remains constant for values of $v$ which are within the coherence bandwidth. Under this model, the singular value decomposition (SVD) of the spectral coherence matrices under $\mathcal{H}_{1}$ within the range (13) is

$$
\mathbf{S}_{\mathbf{y}}(v)=\underbrace{N_{0} \mathbf{I}+g_{k} \tilde{\mathbf{g}}_{k} \tilde{\mathbf{g}}_{k}^{H}}_{\doteq \mathbf{U}_{k} \boldsymbol{\Lambda}_{k} \mathbf{U}_{k}^{H}}
$$

where the eigenvector matrix $\mathbf{U}_{k}$ has been defined as $\mathbf{U}_{k}=$ $\left[\tilde{\mathbf{g}}_{k}, \mathbf{U}_{k}^{\perp}\right]$, and $\boldsymbol{\Lambda}_{k}$ is given as

$$
\boldsymbol{\Lambda}_{k}=\left[\begin{array}{cc}
N_{k} & \mathbf{0} \\
\mathbf{0} & N_{0} \mathbf{I}_{L-1}
\end{array}\right] \text {. }
$$

Because this paper addresses generalized likelihood ratio (GLR) detection with unknown white noise PSD and unknown frequency-selective channel, the set of nuisance parameters for the log-likelihood function (9) are

$$
N_{0}, \quad N_{k}=N_{0}+\gamma_{k} \text { and } \tilde{\mathbf{g}}_{k},
$$

for $k=1, \ldots, K$.

Theorem 1. The Neyman-Pearson optimal frequency-domain GLR detector of the second-order cyclostationary signal (2) over a frequency-selective channel is given by

$$
\frac{1}{\left(\prod_{k=1}^{K} \lambda_{k}\right)^{1 / K}\left(1-\sum_{k=1}^{K} \lambda_{k}\right)^{\alpha}} \stackrel{\mathcal{H}_{1}}{\gtrless} \eta,
$$

where $\eta$ is the detection threshold ${ }^{1}, \lambda_{k}=\lambda_{\max }\left\{\mathbf{B}_{k}\right\}$ with

$$
\mathbf{B}_{k}=\frac{1}{\hat{P}_{T}} \int_{I_{k}} \mathbf{y}(v) \mathbf{y}^{H}(v) d v
$$

\footnotetext{
${ }^{1}$ Setting the detection threshold requires the statistical characterization of the detector under $\mathcal{H}_{0}$. Even though this is in general a hard problem which has only been addressed in some specific situations, one can resort to the Wilks' theorem [22] as a general anlytical tool to set the threshold.
}

$\hat{P}_{T}$ is an estimate of the total received signal power.

Proof. The derivation of (17) involves the maximum likelihood (ML) estimation of the nuisance parameters, i.e., the maximization of $\ln \operatorname{GLR}(\mathbf{y}) \doteq L\left(\mathbf{y} \mid \mathcal{H}_{1}\right)-L\left(\mathbf{y} \mid \mathcal{H}_{0}\right)$ with respect to (16), which is reported in [23] and omitted here due to space limitations.

Here, the matrices $\mathbf{B}_{k}$ in (18) are short-band estimates of the spectral covariance matrix of the received signal normalized by the total received signal power. The entries of these matrices can be seen as samples of the frequency-smoothed cyclic periodogram of the signal.

The interpretation of the detector (17) goes as follows. While the first term of the denominator implements a classical measure of inter-band sphericity (a term that refers to flatness), the second term of the denominator is in charge of measuring the intra-band sphericity, i.e., the lack of spectral correlation. The second term has no influence for $\alpha=0$, whereas its relative importance increases within the excess band. As a whole, the value of the detector increases when high spectral correlation, i.e., high eigenvalue dispersion in $\mathbf{B}_{k}$, is assessed, and/or when the the measured spectrum is far from white.

\section{NUMERICAL RESULTS}

The performance of the proposed detector (17), referred as GLRT- $K$, is evaluated by Monte Carlo simulations using 100,000 realizations per scenario. The transmission is modeled by $M$ QPSK symbols using root raised cosine (RRC) pulses. A tapped delay line channel model [24] with exponentially decaying power profile is considered with mean delay-spread of $\tau \propto 1 / B_{c}$ symbols, such that is remains constant in each realization of $M T$ seconds.

\subsection{Benchmark Detectors}

For comparison, the following detectors are evaluated in all the scenarios. First, the well-known MCSCMD- $K$ proposed by Gardner [10] with $\ell_{0}=1$. Second, the GLR detector not exploiting the frequency-selective nature of the fading channel, i.e., considering $\mathbf{g}(v)=\sqrt{g} \tilde{\mathbf{g}}(v)$ instead of (11). This yields

$$
\frac{1}{\lambda(1-\lambda)^{\alpha}} \stackrel{\mathcal{H}_{1}}{\gtrless} \mathcal{H}_{0} \eta
$$

with $\lambda=\hat{P}_{s} / \hat{P}_{T}$ [23]. It is noted that this corresponds to the GLRT-1, i.e., (17) particularized for $K=1$. This detector is considered to illustrate the lack of robustness in presence of a frequency-selective channel. Third, the LMPIT- $K$, i.e., the Frobenius norm of a normalized version of the autocorrelation matrix

$$
\sum_{k=1}^{K}\left\|\mathbf{B}_{k}\right\|_{F}^{2} \underset{\mathcal{H}_{0}}{\stackrel{\mathcal{H}_{1}}{\gtrless}} \eta .
$$




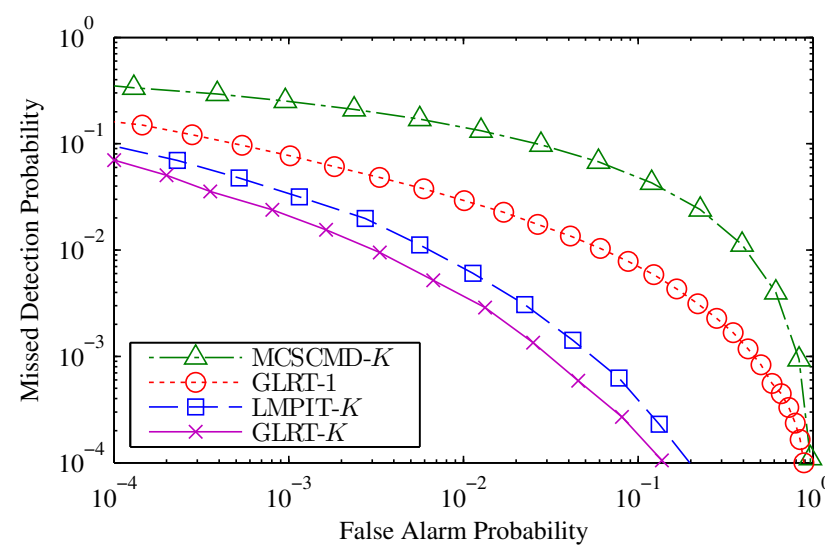

Fig. 1. Complementary ROC with $\tau=1, \alpha=0.8$, SNR $=$ $-0.5 \mathrm{~dB}, M=256$, and $K=8$.

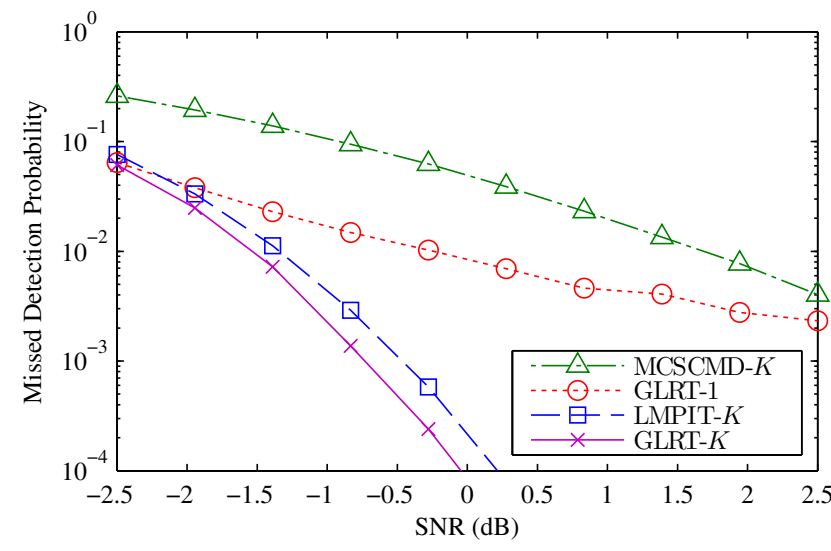

Fig. 2. Missed detection probability versus SNR for a CFAR of 0.05 with $\tau=1, \alpha=0.8, M=256$, and $K=8$.

This detector has been proposed [11,25] and proved [7] to be optimal for close hypotheses, i.e., low-SNR. This is the main reference for comparison, as it assumes identical prior knowledge.

\subsection{Detection Performance}

Fig. 1 depicts the complementary receiver operating characteristics (ROC) when the channel has a mean delay-spread of $\tau=1$ symbol and illustrates the high sensitivity of GLRT-1 in front of an unknown channel response. In contrast, the performance of the proposed GLRT- $K$ detector exhibits a robust behavior in detection performance in front of unknown frequency-selective channels.

The missed detection probability of the detectors versus the SNR is shown in Fig. 2, where the thresholds have been set to satisfy a constant false alarm rate (CFAR) of 0.05 . It is

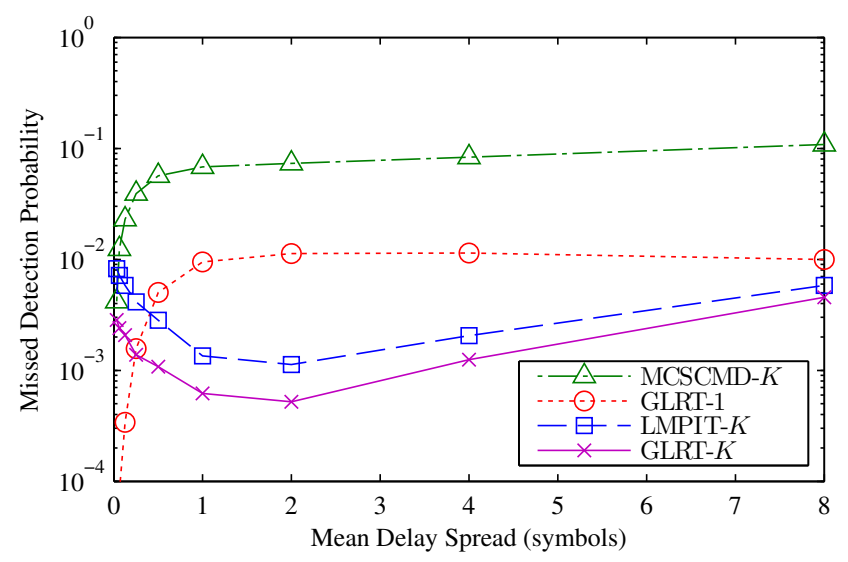

Fig. 3. Missed detection probability versus $\tau$ for a CFAR of 0.05 with $\alpha=0.8, \mathrm{SNR}=-0.5 \mathrm{~dB}, M=256$, and $K=8$.

rapidly observed that the error exponent associated to this error probability, i.e., the scaling of the detection performance with the SNR conditions, is significantly improved by the proposed GLRT- $K$.

Fig. 3 illustrates the main advantage of the proposed detector by showing the missed detection probability versus the mean delay-spread $\tau$, with a CFAR of 0.05 . It is rapidly appreciated that small values of delay-spread cause a significant degradation in the detectors' performance. In contrast, the GLRT- $K$ tolerates higher delay spreads and shows a degradation only when the delay-spread is of the same order as the number of sub-bands, i.e., $K$. Furthermore, the performance of the GLRT- $K$ improves for small values of the delay-spread. This is due to the fact that the GLRT- $K$ is partly sensitive to the lack of sphericity measured by the geometrical mean term in the expression (17). Hence, in contrast to other cyclostationary detectors, the proposed detector does not ignore the stationary spectral component of the signal, which is less flat in the presence of a frequency-selective channel.

Finally, compared to the benchmarking LMPIT- $K$, the proposed GLRT- $K$ provides a systematic gain in the simulated scenarios, whereas the performance of the LMPIT- $K$ approaches that of GLRT- $K$ for asymptotically low-SNR, as expected and confirmed in Fig. 3.

\section{CONCLUSIONS}

In this paper, the GLR detection of a cyclostationary signal is addressed by exploiting the rank-1 structure of small spectral covariance matrices in a novel frequency-domain representation of digital waveforms. By incorporating a $K$ parameterized frequency-selective channel model based on the coherence bandwidth, the proposed GLRT- $K$ is derived. Numerical results have assessed the detection performance and the robustness in front of frequency-selective channels. 


\section{REFERENCES}

[1] E. Axell, G. Leus, E. G. Larsson, and H. V. Poor, "Spectrum sensing for cognitive radio: State-of-the-art and recent advances," IEEE Signal Process. Magazine, vol. 29, no. 3, pp. 101-116, Mar. 2012.

[2] Z. Quan, W. Zhang, S. J. Shellhammer, and A. H. Sayed, "Optimal spectral feature detection for spectrum sensing at very low SNR," IEEE Trans. Commun., vol. 59, no. 1, pp. 201-212, Jan. 2011.

[3] R. Tandra and A. Sahai, "SNR walls for feature detectors," in IEEE Int. Symp. on New Front. in Dyn. Spec. Access, Apr. 2007.

[4] O. Besson, S. Kraut, and L. L. Scharf, "Detection of an unknown rank- one component in white noise," IEEE Trans. Signal Process., vol. 54, no. 7, pp. 2835-2839, Jul. 2006.

[5] R. Zhang, T. J. Lim, Y.-C. Liang, and Y. Zeng, "Multiantenna based spectrum sensing for cognitive radios: A GLRT approach,” IEEE Trans. Commun., vol. 58, no. 1, pp. 84-88, Jan. 2010.

[6] "Detection of rank- $P$ signals in cognitive radio networks with uncalibrated multiple antennas," IEEE Trans. Signal Process., vol. 59, no. 8, pp. 3764-3774, Aug. 2011.

[7] D. Ramirez, J. Via, I. Santamaria, and L. L. Scharf, "Locally most powerful invariant tests for correlation and sphericity of Gaussian vectors," IEEE Trans. Inf. Theory, vol. 59, no. 4, pp. 2128-2141, Apr. 2013.

[8] W. Zhang, H. V. Poor, and Z. Quan, "Frequency-domain correlation: An asymptotically optimum approximation of quadratic likelihood ratio detectors," IEEE Trans. Signal Process, vol. 58, no. 3, pp. 969-979, Mar. 2010.

[9] W. A. Gardner, "Signal interception: A unifying theoretical framework for feature detection," IEEE Trans. Commun., vol. 36, no. 8, pp. 897-906, Aug. 1986.

[10] W. A. Gardner and C. M. Spooner, "Detection and source location of weak cyclostationary signals: simplifications of the maximum-likelihood receiver," IEEE Trans. Commun., vol. 41, no. 6, pp. 905-916, Jun. 1993.

[11] J. Font-Segura, J. Riba, J. Villares, and G. Vazquez, "Quadratic sphericity test for blind detection over timevarying frequency-selective channels," in IEEE Int. Conf. on Acoust., Speech and Signal Process. (ICASSP), May 2013.

[12] G. Huang and J. K. Tugnait, "On cyclostationarity based spectrum sensing under uncertain Gaussian noise,"
IEEE Trans. Signal Process., vol. 61, no. 8, pp. 20422054, Apr. 2013.

[13] R. Tandra and A. Sahai, "SNR walls for signal detection,” IEEE J. Sel. Topics Signal Process., vol. 2, no. 1, pp. 4-17, Feb. 2008.

[14] J. Riba, J. Villares, and G. Vazquez, "A nondata-aided SNR estimation technique for multilevel modulations exploiting signal cyclostationarity," IEEE Trans. Signal Process., vol. 58, no. 11, pp. 5767-5778, Nov. 2010.

[15] M. Sadler and A. V. Dandawate, "Nonparametric estimation of the cyclic cross spectrum," IEEE Trans. Inf. Theory, vol. 44, no. 1, pp. 351-358, Jan. 1998.

[16] P. J. Schreier and L. L. Scharf, Statistical Signal Processing of Complex-Valued Data. The Theory of Improper and Noncircular Signals. Cambridge University Press, 2010.

[17] W. A. Gardner, Statistical Spectral Analysis: A Nonprobabilistic Theory. Prentice-Hall, 1986.

[18] E. Serpedin, F. Panduru, I. Sari, and G. B. Giannakis, "Bibliography on cyclostationarity," Signal Process., vol. 85, no. 12, pp. 2233-2303, 2005.

[19] W. A. Gardner, A. Napolitano, and L. Paura, "Cyclostationarity: Half a century of research," Signal Process. vol. 86, no. 4, pp. 639-697, Apr. 2006.

[20] D. R. Brillinger, Time Series: Data Analysis and Theory. San Francisco: Holden-Day, 1981, (Section 4.4: asymptotic distribution of the finite Fourier transform).

[21] A. V. Dandawate and G. B. Giannakis, "Statistical tests for presence of cyclostationarity," IEEE Trans. Signal Process., vol. 42, no. 9, pp. 2355-2369, Sep. 1994.

[22] S. M. Kay, Fundamentals of Statistical Signal Processing: Detection Theory. New York: Prentice-Hall, 1998, vol. 2 .

[23] J. Riba, J. Font-Segura, J. Villares, and G. Vazquez, "Frequency-domain GLR detection of a second-order cyclostationary signal over fading channels," IEEE Tran. Signal Process., 2014, (in press).

[24] E. Biglieri, J. Proakis, and S. Shamai, "Fading channels: information-theoretic and communications aspects," IEEE Trans. Inf. Theory, vol. 44, no. 6, pp. 26192692, Oct. 1998.

[25] A. Leshem and A.-J. V. der Veen, "Multichannel detection of Gaussian signals with uncalibrated receivers," IEEE Signal Process. Lett., vol. 8, no. 4, pp. 120-122, Apr. 2001. 